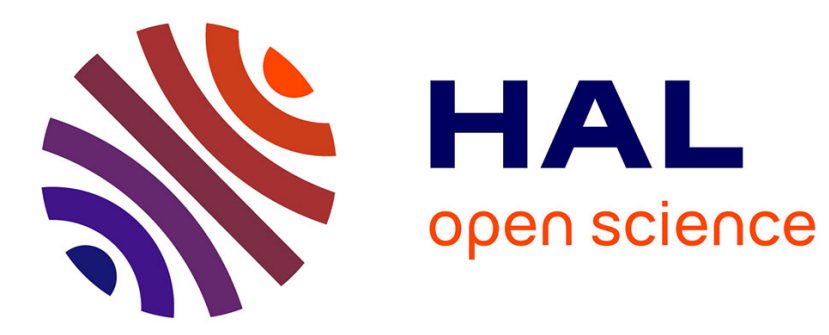

\title{
Nonlinear spectral broadening of femtosecond pulses in solid-core photonic bandgap fibers
}

\author{
V. Pureur, J.M. Dudley
}

\section{To cite this version:}

V. Pureur, J.M. Dudley. Nonlinear spectral broadening of femtosecond pulses in solid-core photonic bandgap fibers. Optics Letters, 2010, 35 (16), pp.2813-2815. 10.1364/OL.35.002813 . hal-00517180

\author{
HAL Id: hal-00517180 \\ https://hal.science/hal-00517180
}

Submitted on 4 May 2021

HAL is a multi-disciplinary open access archive for the deposit and dissemination of scientific research documents, whether they are published or not. The documents may come from teaching and research institutions in France or abroad, or from public or private research centers.
L'archive ouverte pluridisciplinaire HAL, est destinée au dépôt et à la diffusion de documents scientifiques de niveau recherche, publiés ou non, émanant des établissements d'enseignement et de recherche français ou étrangers, des laboratoires publics ou privés. 


\title{
Nonlinear spectral broadening of femtosecond pulses in solid-core photonic bandgap fibers
}

\author{
Vincent Pureur* and John M. Dudley \\ Institut FEMTO-ST, Département d'Optique P. M. Duffieux, CNRS UMR 6174, \\ Université de Franche-Comté, 25030 Besançon, France \\ *Corresponding author: vincent.pureur@femto-st.fr
}

\begin{abstract}
We study nonlinear spectral broadening and supercontinuum generation mechanisms in two-dimensional solidcore photonic bandgap fibers. Using rigorous frequency-domain numerical simulations, we determine how the spectral characteristics are influenced by the strong frequency dependence of the effective area, dispersion, and confinement losses. We also investigate soliton stabilization and the conditions under which efficient nonlinear spectral energy transfer is possible across high attenuation between adjacent bandgaps. Our results provide insight into recent experiments.
\end{abstract}

Two-dimensional solid-core photonic bandgap (PBG) fibers are a class of photonic crystal fibers (PCFs) in which light propagates in a solid core as a result of PBG guidance $[1,2]$. In contrast to index-guiding fibers, PBG fibers can support propagation across several distinct spectral windows corresponding to bandgaps (BGs) of a different order [3]. The frequency dependence of the BG guidance characteristics is very strong near the PBG edges and, thus, is expected to significantly influence broadband nonlinear propagation [4]. However, although there have been several recent experimental and numerical studies of nonlinear broadening in a solid-core structure over moderate wavelength spans [5-7], there have been no detailed studies of precisely how the different PBG characteristics impact on broadband spectral generation over a significant fraction of the PBG window. In this Letter, we use a full frequency-domain approach to simulate femtosecond pulse supercontinuum (SC) generation and consider in detail for the first time (to our knowledge) how the strong frequency dependence of the effective area, dispersion, and losses all significantly influence the output characteristics. Our simulations successfully reproduce two recently observed experimental effects of soliton stabilization and nonlinear spectral energy transfer across adjacent PBGs.

We consider a structure, such as in [2], with a low-index silica core surrounded by four high-index inclusion rings (Cargille oil, $n=1.6$ at $\lambda=589.3 \mathrm{~nm}$ ), with pitch $3.10 \mu \mathrm{m}$ and diameter $1.23 \mu \mathrm{m}$. We use the well-established multipole method (fully described in [8]) to calculate the (non) linear guidance properties (assumed single mode). For both the silica and inclusion rods, we include material dispersion but neglect material loss [9]. Figure 1 plots the results as a function of the normalized frequency $(V$ parameter) of an isolated high-index rod [10]. The top panel shows the linear properties: confinement loss (left axis) and group-velocity dispersion [(GVD) right axis]. The bottom panel plots the effective area $A_{\text {eff }}$ (left axis) and the nonlinear parameter $\gamma$ (right axis) [11]. We show results for the first four PBGs whose transmission windows and zero-dispersion wavelengths (ZDWs) are, respectively: $\sim 2600-1090 \mathrm{~nm}$ and $1287 \mathrm{~nm}$ (PBG1), $1090-680 \mathrm{~nm}$ and $790 \mathrm{~nm}$ (PBG2), $680-480 \mathrm{~nm}$ and $570 \mathrm{~nm}$ (PBG3), and $480-400 \mathrm{~nm}$ and $458 \mathrm{~nm}$ (PBG4). The lowest-order mode cut-off frequencies of an isolated inclusion (vertical dashed lines) delimit approximately the different PBGs [10]. Note that the guidance properties are predominantly determined by the PBG structure and are typical of those to be found in the literature and used in recent experiments [5-7]. To illustrate the challenges posed by modeling propagation in solid-core PBG fibers, Fig. 1 also plots $\mathrm{A}_{\text {eff }}$ and $\gamma$ (dashed curves) for an indexguiding PCF with a silica core surrounded by air holes (dimensions as above). The major difference compared to index-guiding PCFs is that the PBG fiber effective area, loss, and GVD undergo dramatic variations near the PBG edges, where the guided mode couples energy to the cladding modes.

The strong frequency dependence of guidance properties significantly influences nonlinear propagation as spectral broadening develops toward the PBG edges.



Fig. 1. (Color online) Top axes: loss (red, left) and GVD (black, right) as a function of normalized frequency $V$. Bottom axes: effective area (blue, left) and nonlinear parameter $\gamma$ (green, right). The dashed curves show results for an indexguiding $\mathrm{PCF}$. 
Thus, although approximate models may be suitable to study spectral evolution near the BG center [5], more rigorous simulations require full frequency-domain modeling. To study this in detail, we use the Laegsgaard approach (Eq. 33 of [11]) and with Raman parametrization, as in [12]. This approach has been verified by experiments and independent simulations [11], and allows an approximation-free treatment of the frequencydependent loss, dispersion, and nonlinearity within each PBG and across adjacent BGs. Note that related approximate methods are unsatisfactory [4,13].

We first simulate SC generation where spectral content is confined within only PBG1. Figure 2(a) plots the guidance properties and shows how the wavelengths of zero GVD, minimum loss, and $A_{\text {eff }}$ can be different in $P B G$ fibers. In Figs. 2(b) and 2(c), we plot simulated spectra after $50 \mathrm{~cm}$ propagation for $100 \mathrm{~kW}, 50$ fs Gaussian input pulses at two pump wavelengths. We see significant differences in spectral characteristics, depending on which particular frequency-dependent parameters are modeled, and we plot results for three different cases: (i) the realistic case including full frequency dependence of both $\mathrm{A}_{\text {eff }}(\omega)$ and loss $\alpha(\omega)$ (red solid curve); (ii) an approximation including full frequency dependence of $\mathrm{A}_{\text {eff }}(\omega)$ but neglecting loss, i.e., $\alpha(\omega)=0$ (green short-dashed curve); and (iii) an approximation where $\mathrm{A}_{\text {eff }}\left(\omega_{0}\right)$ is constant evaluated only at the pump but frequency-dependent loss $\alpha(\omega)$ is still included (blue long-dashed curve).

These three approaches to modeling yield very significant differences that vary depending on the pump position relative to the $\mathrm{ZDW}$. We highlight two specific cases: [Fig. 2(b)] pumping at $1290 \mathrm{~nm}$, where soliton fission and dispersive wave (DW) generation yield a broad SC over

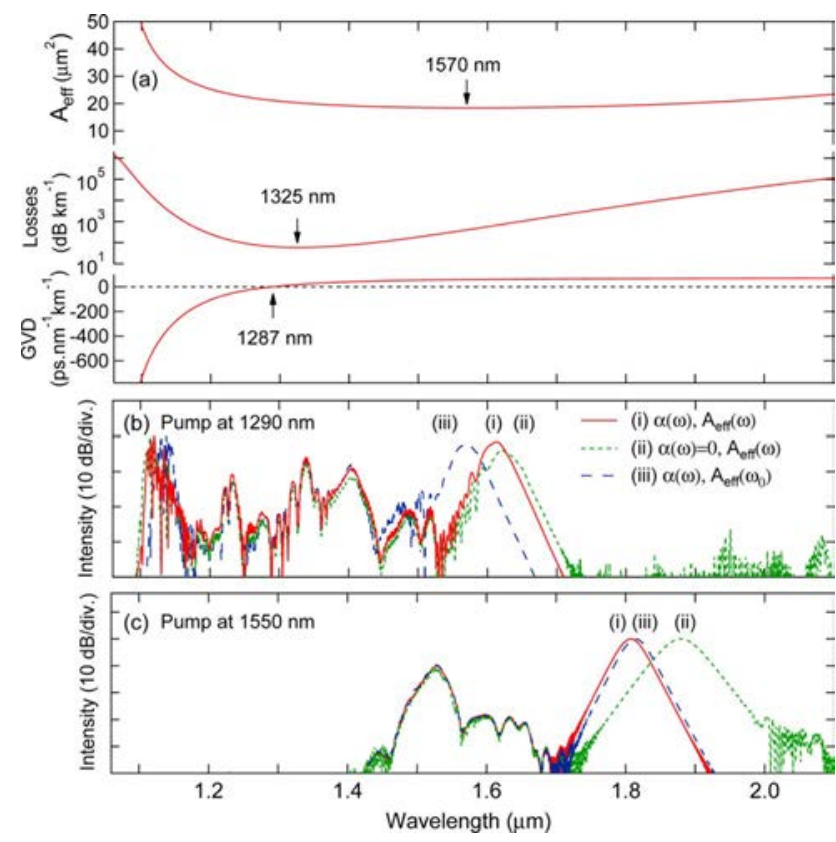

Fig. 2. (Color online) (a) PBG1 characteristics compared with SC simulations pumping at (b) $1290 \mathrm{~nm}$ and (c) $1550 \mathrm{~nm}$. Results show (i) fully realistic modeling of frequency-dependent effective area $\mathrm{A}_{\text {eff }}(\omega)$ and loss $\alpha(\omega)$ (red solid curve, seven rings); (ii) approximation with frequency-dependent $\mathrm{A}_{\text {eff }}(\omega)$ but no loss, i.e., $\alpha(\omega)=0$ (green short-dashed curve); and (iii) approximation with constant $\mathrm{A}_{\text {eff }}\left(\omega_{0}\right)$ and frequencydependent loss $\alpha(\omega)$ (blue long-dashed curve).
$1.1-1.6 \mu \mathrm{m}$, and [Fig. 2(c)] pumping further from the ZDW at $1550 \mathrm{~nm}$, where the dynamics predominately involve the Raman self-frequency shift of a single soliton pulse. As might be expected, neglecting the frequency-dependent loss [curve (ii)] predicts an overestimated spectral width in both cases [Figs. 2(b) and 2(c)], and the degree of overestimate increases as the spectrum extends closer to the band edges in Fig. 2(c).

The effect of the frequency-dependent effective area on nonlinear propagation in PBG fibers can be very different to the case of index-guiding PCFs. For the $1550 \mathrm{~nm}$ pump in Fig. 2(c), the effective area (minimal at $1570 \mathrm{~nm}$ ) increases toward the longer wavelength edge of the SC, a situation identical to that of standard fibers [13]. In this case, the realistic simulations in curve (i) accurately model the decrease in nonlinearity and frequency shift experienced by the solitons that shift to longer wavelengths relative to the case of constant $\mathrm{A}_{\text {eff }}$ in (iii). In contrast, pumping at $1290 \mathrm{~nm}$ closer to the ZDW in Fig. 2(b) yields a propagation scenario not seen at all in standard fibers with the effective area decreasing with increasing wavelength across the SC bandwidth. Here, the realistic simulations that include this variation in (i) predict an increased soliton self-frequency shift (SSFS) when compared to simulations in (iii) assuming a constant (and higher) value for $\mathrm{A}_{\text {eff }}$.

These results show that failure to include the frequency dependence of the PBG characteristics will lead to severe inaccuracies when modeling experiments. They also provide insight into particular effects introduced by the frequency dependence of the nonlinearity and loss. One important consequence is that interpreting nonlinear spectral broadening mechanisms in PBG fibers depends critically on how the spectral span varies relative to regions of increasing or decreasing nonlinearity and dispersion. This is a major result of this Letter and places fundamentally different constraints on modeling when compared to standard fibers.

Our approach also allows us to interpret the novel physics associated with recent experimental results. We first consider the challenging case of modeling propagation in multiple PBGs simultaneously, as reported in [7]. Note that our modeling allows direct approximationfree simulation without coupled mode decomposition. Specifically, we simulate $100 \mathrm{~kW}, 50 \mathrm{fs}$ pulses injected into the anomalous regime of PBG2 (ZDW at $790 \mathrm{~nm}$ ), with full $\mathrm{A}_{\text {eff }}(\omega)$ and $\alpha(\omega)$. Figure $3(\mathrm{a})$ plots the spectra after $50 \mathrm{~cm}$ propagation for pump wavelengths of $830 \mathrm{~nm}$ and $880 \mathrm{~nm}$, as well as fiber transmission (dashed curve) across PBG2 and PBG3. These results reproduce very well the features seen in experiments and, moreover, provide significant insight into the physics of PBG fibers propagation in this regime. For example, Fig. 3(a) reveals a strong dependence of the short wavelength DW amplitude on pump wavelength, because longer pump wavelengths phase match to shorter wavelength DWs, which see more band-edge attenuation. Moreover, although the energy transfer across the interband attenuation region may seem surprising, we can understand this physically by inspecting the initial propagation dynamics in detail as shown in Fig. 3(b) (right) for the $830 \mathrm{~nm}$ pump. This shows how the DW transfer occurs only over the first few millimeters of propagation, and because the 


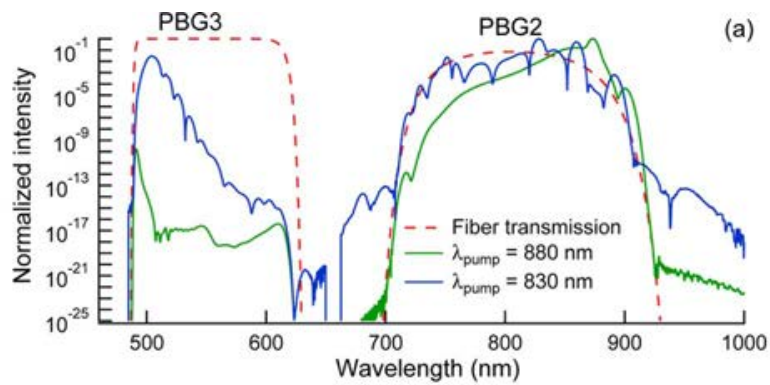

(b)
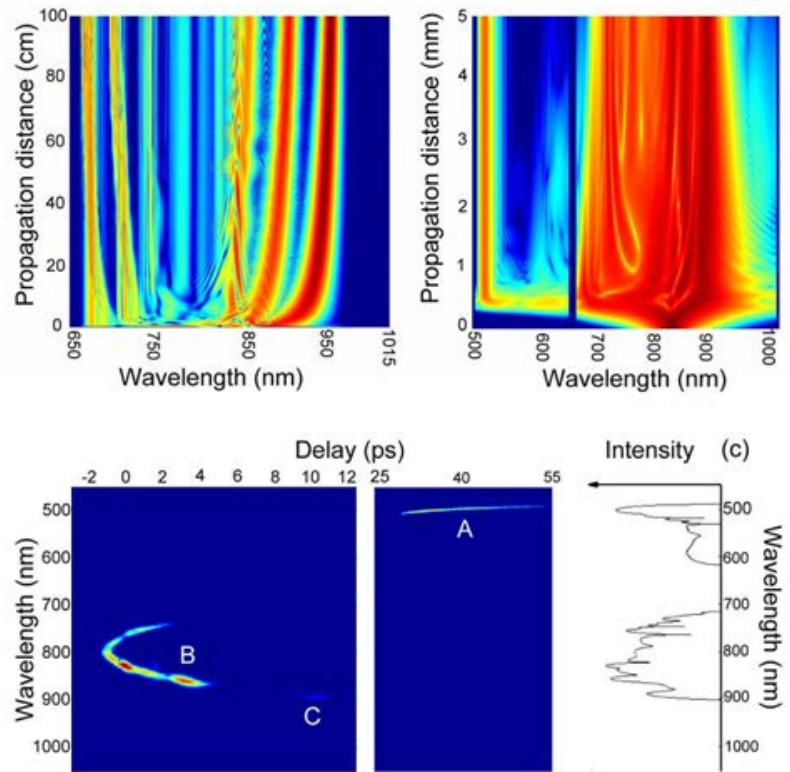

Fig. 3. (Color online) (a) Output spectra after $50 \mathrm{~cm}$ propagation for pump wavelengths as shown. (b) SC evolution in PBG2 over fiber length of $1 \mathrm{~m}$ with $\alpha=0$ (left) and simulated SC evolution in PBG2 and PBG3 over a propagation distance of $5 \mathrm{~mm}$ ( $\lambda_{\text {pump }}=830 \mathrm{~nm}, P_{\text {in }}=100 \mathrm{~kW}$ ) (right). (c) Output spectrogram correlating DW (A) and solitons (B) and (C).

loss integrated along the nonlinear length of the input pulse $\left(\mathrm{L}_{\mathrm{NL}} \sim 0.8 \mathrm{~mm}\right.$ ) is relatively low at $\sim 2.6 \mathrm{~dB}$, it is easy to see how the initial spectral broadening phase can still transfer significant energy into the adjacent PBG to seed DW generation. Of course, once generated in low-loss regions of PBG3, the short-wavelength components see much lower attenuation.

Figure 3(c) yields further insight by plotting the output spectrogram, correlating the DW (A) and solitons (B) and (C) components with the spectrum. This reveals how long wavelength band edge loss can play a role in attenuating the amplitude of soliton C. We also note large temporal separation between the pump and DW spectral components arising from the very different dispersion properties of the two PBGs. Indeed the group velocity walk-off between the $500 \mathrm{~nm} \mathrm{DW}$ and the $830 \mathrm{~nm}$ pump is $\sim 80 \mathrm{ps} / \mathrm{m}, 3-4$ times larger than walk-off seen in in- dex-guiding PCFs. This is a particular feature of propagation in PBG fibers that would need to be accurately taken into account for applications requiring temporal synchronization. In addition to multiple PBG propagation, our simulations [Fig. 3(b) (left)] also reproduce the recently observed SSFS cancellation of the first ejected soliton close to the red edge of a single PBG [14]. Significantly, we note that these results were obtained including only frequency-dependent nonlinearity and dispersion, providing insight into the minor role played by spectral filtering for this regime.

The main conclusion of our results is that the strong frequency dependence of the nonlinearity, dispersion, and loss must all be included to accurately model experiments in solid-core PBG fibers. Moreover, we have shown how the frequency dependence of the effective area can act to modify the spectrum in a way that is not seen in standard fiber. Our modeling has also been used to provide insight into recent experiments.

We thank A. Judge, B. Kuhlmey, and J. C. Travers for discussions. Financial support from the Agence Nationale pour la Recherche, projects MANUREVA ANR-08-SYSC019 and IMFINI ANR-09-BLAN-0065, is acknowledged.

\section{References}

1. A. Argyros, T. A. Birks, S. G. Leon-Saval, C. M. B. Cordeiro, F. Luan, and P. St. J. Russell, Opt. Express 13, 309 (2005).

2. V. Pureur, L. Bigot, G. Bouwmans, Y. Quiquempois, M. Douay, and Y. Jaouen, Appl. Phys. Lett. 92, 061113 (2008).

3. V. Pureur, A. Bétourné, G. Bouwmans, L. Bigot, A. Kudlinski, K. Delplace, A. Le Rouge, Y. Quiquempois, and M. Douay, Fiber Integr. Opt. 28, 27 (2009).

4. J. M. Dudley, G. Genty, and S. Coen, Rev. Mod. Phys. 78, 1135 (2006).

5. A. Fuerbach, P. Steinvurzel, J. A. Bolger, A. Nulsen, and B. J. Eggleton, Opt. Lett. 30, 830 (2005).

6. B. Kibler, T. Martynkien, M. Szpulak, C. Finot, J. Fatome, J. Wojcik, W. Urbanczyk, and S. Wabnitz, Opt. Express 17, 10393 (2009).

7. A. Cerqueira, C. M. B. Cordeiro, F. Biancalana, P. J. Roberts, H. E. Hernandez-Figueroa, and C. H. Brito Cruz, Opt. Lett. 33, 2080 (2008).

8. T. P. White, B. T. Kuhlmey, R. C. McPhedran, D. Maystre, G. Renversez, C. M. de Sterke, and L. C. Botten, J. Opt. Soc. Am. B 19, 2322 (2002).

9. P. D. Rasmussen, J. Laegsgaard, and O. Bang, Opt. Express 16, 4059 (2008).

10. N. M. Litchinitser, S. C. Dunn, B. Usner, B. J. Eggleton, T. P. White, R. C. McPhedran, and C. M. de Sterke, Opt. Express 11, 1243 (2003).

11. J. Laegsgaard, Opt. Express 15, 16110 (2007).

12. G. P. Agrawal, Nonlinear Fiber Optics (Academic, 2007).

13. B. Kibler, J. M. Dudley, and S. Coen, Appl. Phys. B 81, 337 (2005).

14. A. Bétourné, A. Kudlinski, G. Bouwmans, O. Vanvincq, A. Mussot, and Y. Quiquempois, Opt. Lett. 34, 3083 (2009). 